\title{
Análisis de las lesiones causadas por el tránsito sufridas por ciclistas en México
}

\author{
Victoria Alejandra Muro-Báez', M. Eulalia Mendoza-García², Juan Daniel Vera-López³ \\ y Ricardo Pérez-Núñez \\ ${ }^{1}$ Estudiante de la Licenciatura en Sociología; ' Licenciada en Sociología; ${ }^{3}$ Maestro en Ciencias de la Salud; ${ }^{4}$ Doctor en Ciencias de la Salud Pública. \\ Secretariado Técnico del Consejo Nacional para la Prevención de Accidentes, Secretaría de Salud México, Ciudad de México, México
}

\section{Resumen}

Para analizar las lesiones fatales y no fatales de tránsito ocurridas en ciclistas, y documentar el uso de casco buscando informar las políticas públicas de transporte sostenible, se realizó un análisis descriptivo de cuatro fuentes de información secundaria a escala nacional: mortalidad, egresos hospitalarios de la Secretaría de Salud, Sistema de Registro de Atención por Violencia o Lesión(SIS-17) y Encuesta Nacional de Salud y Nutrición 2012 (ENSANUT). Solo las dos últimas fuentes documentan el uso de casco. Salvo la ENSANUT, la información fue de 2014. Fallecieron 190 ciclistas y 392 fueron hospitalizados; la cabeza fue la región anatómica con más frecuencia afectada (63\% y 32\%, respectivamente). Solo el $0.75 \%$ de los 667 casos registrados en SIS-17 utilizaba casco al momento de la colisión, y el 24\% sufrió lesiones en la cabeza. De los 165,348 ciclistas con lesiones no fatales de ENSANut, menos del 10\% utilizaba casco, el $24 \%$ se lesionó la cabeza o el cuello, y más de 16,000 reportaron consecuencias permanentes. Mientras se promueve una infraestructura segura para ciclistas, el uso de casco podría reducir la frecuencia y la gravedad de las lesiones de cabeza en el corto plazo en lo que el uso de bicicleta se populariza como medio de transporte, ofreciendo «seguridad en números».

PALABRAS CLAVE: Accidentes de tránsito. Ciclistas. Casco. Lesiones.

\begin{abstract}
With the objective of analyzing fatal and non-fatal road traffic injuries in cyclists and to document helmet use in this road user to inform sustainable mobility policies, a descriptive analysis of four secondary official information sources was conducted at the national level: mortality, Ministry of Health's hospital discharges, Unintentional and Violence Registry System (SIS-SS-17-P) and the 2012 National Health and Nutrition Survey (ENSANUT). Only SIS-SS-17-P and ENSANUT document helmet use. Except for ENSANUT information analyzed is of 2014.A total of 190 cyclists died in Mexico during 2014 and 392 were hospitalized; head was the anatomical region most frequently affected (63\% and 32\%, respectively). Only $0.75 \%$ of the 667 cases registered in SIS-17 reported helmet use and 24\% suffered head injuries. Of the 165,348 non-fatally injured cyclists from ENSANUT $<10 \%$ used helmet, $24 \%$ had head injuries and more than 16,000 suffered permanent injuries. Whereas cyclist-friendly infrastructure is an effective intervention to prevent injuries in the long term, helmet use could potentially reduce the frequency and severity of head injuries in the short run while bicycle use widespread as a means of transportation providing "safety in numbers".
\end{abstract}

KEY WORDS: Road traffic injuries. Cyclists. Helmet. Injuries.

\author{
Correspondencia: \\ Ricardo Pérez-Núñez \\ Guadalajara, 46, tercer piso \\ Col. Roma Norte, Del. Cuauhtémoc \\ C.P. 06700 , Ciudad de México, México \\ E-mail: ricardo.perez@insp.mx
}

Fecha de recepción: 20-07-2016

Fecha de aceptación: 08-08-2016

DOI:10.24875/GMM.17002632
Gac Med Mex. 2017;153:653-661

Contents available at PubMed www.gacetamedicademexico.com 


\section{Introducción}

De acuerdo con la Organización Mundial de la Salud, cada año 1.25 millones de muertes son atribuidas a lesiones causadas por el tránsito (LCT), y de estas un $4 \%$ corresponde a ciclistas, lo que representa que anualmente alrededor de 50,000 ciclistas fallezcan en todo el mundo por esta causa. Se ha documentado que en algunos países de bajos y medianos ingresos este porcentaje podría ser mayor ${ }^{1}$. Los ciclistas son considerados usuarios vulnerables de las vialidades, ya que no están protegidos por una estructura física, lo que los expone a sufrir lesiones más graves en caso de presentarse un evento de tránsito o incluso de morir al momento de sufrir una colisión. Esto es relevante porque los ciclistas comparten la vía pública con automóviles privados y vehículos de transporte de carga o pasajeros que se desplazan rápidamente. En contextos donde el uso de la bicicleta no es muy común o en los que recientemente está siendo impulsado, los ciclistas suelen ser poco visibles para los conductores de vehículos motorizados ${ }^{2}$.

Recientemente, en México se han impulsado políticas públicas encaminadas a fomentar una movilidad más sustentable. Una de ellas es el uso de la bicicleta como medio de transporte en distintas partes del territorio nacional, a través de sistemas de bicicletas públicas. Por ejemplo, el sistema $\mathrm{ECOBICl}^{3}$ en la Ciudad de México, implementado desde febrero de 2010, que actualmente cuenta con 444 ciclo-estaciones, más de 6000 bicicletas y más de 100,000 usuarios diariamente, cubriendo un área de $35 \mathrm{~km}^{2}$; el sistema $\mathrm{MIBICl}^{4}$ en la ciudad de Guadalajara, desde diciembre de 2014, que actualmente cuenta con 116 ciclo-estaciones (ubicadas en las zonas centro de Guadalajara y Zapopan) y 1160 bicicletas; la prueba piloto del sistema de bicicletas públicas SmartBike $^{5}$ en la ciudad de Puebla, que desde noviembre de 2013 y hasta septiembre de 2015 contempló un cuadrante de 5.6 kilómetros del centro histórico, con seis ciclo-estaciones y 81 bicicletas; y la implementación del sistema de bici pública Huizi ${ }^{6}$ de la ciudad de Toluca, el cual cuenta con 26 ciclo-estaciones alrededor de 218 manzanas de la zona centro de la ciudad y 350 bicicletas. En conjunto, según el Instituto de Políticas para el Transporte y el Desarrollo, en las 30 ciudades más grandes del país se cuenta con un registro nacional de 477 kilómetros de infraestructura ciclista, equivalentes a $0.8 \mathrm{~km}$ por cada 100,000 habitantes, de los cuales el $20 \%$ son ciclovía unidireccional, el $20 \%$ ciclocarriles, el $58 \%$ ciclovía bidireccional y el $2 \%$ carril compartido (transporte público y bicicleta) $)^{7}$.

El incremento del uso de la bicicleta y la inserción de los sistemas de bicicletas públicas en las zonas urbanas motivan el presente estudio que busca hacer un diagnóstico en México de los principales daños a la salud experimentados por los ciclistas como resultado de las LCT. De igual forma, se explora el uso de casco como dispositivo de seguridad en este grupo poblacional. Consideramos que este análisis permitirá dar seguimiento a estas políticas públicas de movilidad y establecer acciones específicas que garanticen que dicha movilidad se desarrolle en un contexto de seguridad vial para estos usuarios vulnerables.

\section{Métodos}

Se trata de un estudio transversal que utiliza fuentes secundarias a escala nacional para identificar el perfil sociodemográfico de los ciclistas con lesiones fatales y no fatales ocurridas en su tránsito por la vía pública, así como el tipo de lesión más frecuente. Para ello se utilizaron cuatro fuentes de información:

- La base completa de mortalidad de 2014, generada conjuntamente por la Secretaría de Salud y el Instituto Nacional de Estadística y Geografía ${ }^{8}$.

- La base de egresos hospitalarios de 2014 de la Secretaría de Salud, que concentra alrededor del $50 \%$ de los egresos hospitalarios del país ${ }^{9}$.

- La información de 2014 del sistema estadístico «SIS-SS-17-P Hoja de Registro de Atención por Violencia y/o Lesión», que opera principalmente en unidades de la Secretaría de Salud ${ }^{10}$.

- La Encuesta Nacional de Salud y Nutrición 2012 (ENSANut-2012), que es representativa del país y que fue elaborada por el Instituto Nacional de Salud Pública"1.

Para fines de este estudio se definió como ciclista a todos los lesionados registrados y clasificados en los siguientes códigos de la Clasificación Estadística Internacional de Enfermedades y Problemas Relacionados con la Salud, en su décima revisión (CIE-10): V10 (.3-.9), V11 (.03-.09), V12 (.3-.9), V13 (.3-.9), V14 (.3-.9) y V19 $(.4-.6)^{12}$.

Se realizó un análisis descriptivo de las principales variables de interés para este trabajo con ayuda de STATA-1 $3^{\circledR}$. La tabla 1 muestra la información de utilidad disponible para cada fuente de información. Es importante hacer notar que, de estas cuatro fuentes, solo la ENSANut-2012 y el SIS-SS-17 registran datos 
Tabla 1. Variables disponibles, según fuente de información

\begin{tabular}{|c|c|c|}
\hline Fuente de información & Variables de interés & Observaciones \\
\hline Mortalidad 2014 & $\begin{array}{l}\text { Sexo, edad, aseguramiento médico, habla lengua } \\
\text { indígena, estado civil, escolaridad, tipo de colisión, } \\
\text { nacionalidad, lugar donde ocurrió la defunción, } \\
\text { recibió atención médica antes de fallecer, ocurrió en el } \\
\text { desempeño laboral. }\end{array}$ & $\begin{array}{l}\text { Para la descripción de la región } \\
\text { anatómica, se consideró la afección } \\
\text { principal. }\end{array}$ \\
\hline $\begin{array}{l}\text { Egresos hospitalarios de la Secretaría } \\
\text { de Salud de } 2014\end{array}$ & $\begin{array}{l}\text { Sexo, edad, aseguramiento médico, habla lengua } \\
\text { indígena, tipo de colisión, motivo del egreso, primera vez } \\
\text { o subsecuente, días de estancia intrahospitalaria. }\end{array}$ & $\begin{array}{l}\text { Para la descripción de la región } \\
\text { anatómica, se consideró la afección } \\
\text { principal. }\end{array}$ \\
\hline $\begin{array}{l}\text { Sistema estadístico "SIS-SS-17-P } \\
\text { Hoja de Registro de Atención por } \\
\text { Violencia y/o Lesión" de } 2014\end{array}$ & $\begin{array}{l}\text { Sexo, edad, aseguramiento médico, escolaridad, tipo } \\
\text { de colisión, si el lesionado estaba bajo los efectos del } \\
\text { alcohol u otra droga, discapacidad preexistente, si recibió } \\
\text { atención médica prehospitalaria y tiempo transcurrido } \\
\text { entre esta atención y el arribo al hospital, destino después } \\
\text { de la atención recibida, condición de embarazo, sucedió } \\
\text { en día festivo, servicio que otorgó la atención, tiempo de } \\
\text { estancia en el servicio de urgencias y uso de casco. }\end{array}$ & $\begin{array}{l}\text { Para la descripción de la región } \\
\text { anatómica, se consideró la afección } \\
\text { principal. }\end{array}$ \\
\hline $\begin{array}{l}\text { Encuesta Nacional de Salud y } \\
\text { Nutrición } 2012\end{array}$ & $\begin{array}{l}\text { Sexo, edad, aseguramiento médico, habla lengua } \\
\text { indígena, estado civil, escolaridad, estrato de residencia, } \\
\text { si el lesionado estaba bajo los efectos del alcohol u otra } \\
\text { droga, si recibió atención (médica formal o no formal } \\
\text { comocurandero, yerbero, huesero, sobador quiropráctico, } \\
\text { etc.), uso de casco y consecuencias permanentes } \\
\text { derivadas de las lesiones sufridas. }\end{array}$ & $\begin{array}{l}\text { La región anatómica se dividió en } \\
\text { grandes categorías que no se pueden } \\
\text { desagregar para su comparación. } \\
\text { El tipo de lesión y su región anatómica } \\
\text { no fue documentado en menores de } 10 \\
\text { años. }\end{array}$ \\
\hline
\end{tabular}

sobre la utilización del casco al momento de sufrir las lesiones. Se reportan frecuencias y porcentajes para las variables categóricas, y media y desviación estándar para las variables continuas. Para evaluar la relación entre el uso de casco y la presencia de lesiones en cabeza, cara y cuello, se realizó la prueba de ji al cuadrado de Pearson para igualdad de proporciones, empleando la prueba exacta de Fisher cuando los valores esperados fueron menores de 5 .

Dado que se realizó un análisis secundario de bases de datos oficiales, sin identificadores individuales, este estudio no tuvo riesgo para ningún participante, por lo que no fue necesario solicitar aprobación por parte de algún comité de ética.

\section{Resultados}

Según los datos de mortalidad, durante 2014 fallecieron 190 ciclistas en el país, lo que representa el $1.20 \%$ del total de personas que fallecieron por LCT en el país. De ellos, solo seis fueron mujeres (3.16\%). La edad promedio fue de 47.67 años (con una desviación estándar de 20.51) y las categorías de edad más afectadas fueron la de 60 y más años y la de 40 a 49 años, con el 31.05 y el $19.47 \%$ de los casos, respectivamente. La mayoría de los ciclistas fallecieron en la vía pública $(63.16 \%)$, y el $28.42 \%$ falleció en una unidad médica pública. En este sentido llama la atención que solo el $32.11 \%$ de los ciclistas fallecidos recibió algún tipo de atención médica antes de morir. Es importante destacar que en el $16.84 \%$ de los casos (32 ciclistas) el evento ocurrió en el desempeño de su trabajo. En la tabla 2 se presenta más información con detalle.

La cabeza fue la región anatómica más frecuentemente afectada en los ciclistas fallecidos, reportándose en el $63.16 \%$ de los casos. El tórax fue la segunda región anatómica en importancia, con un $12.11 \%$ de los ciclistas, seguida de «múltiples regiones" con un $11.58 \%$ (Tabla 3). Ninguno de los fallecidos durante 2014 presentó lesiones en los miembros superiores, los tobillos o los pies.

Los datos de egresos hospitalarios de 2014 revelan que hay alrededor de dos ciclistas hospitalizados, tan solo en la Secretaría de Salud, por cada persona que fallece, considerando que de los 392 egresos registrados el $95.66 \%$ fueron de primera vez y el resto subsecuentes. Los ciclistas representaron el $1.49 \%$ del total de LCT. El grupo de edad con más hospitalizaciones fue el de 10 a 19 años, con un $25.77 \%$, seguido de los grupos de 20 a 29 años y de 30 a 39 años, con un 17.35 y un $15.31 \%$, respectivamente. Se puede observar que la gran mayoría de los lesionados colisionaron contra un automóvil o camioneta (59.18\%). 
Tabla 2. Análisis descriptivo de los ciclistas lesionados por el tránsito en México

\begin{tabular}{|c|c|c|c|c|c|c|c|c|}
\hline \multirow[t]{2}{*}{ Variables de interés } & \multicolumn{2}{|c|}{ Mortalidad 2014} & \multicolumn{2}{|c|}{ Egresos 2014} & \multicolumn{2}{|c|}{ SIS-17 2014} & \multicolumn{2}{|c|}{ ENSANut 2012} \\
\hline & $N=190$ & $\%$ & $\mathrm{~N}=392$ & $\%$ & $N=667$ & $\%$ & $N=165,348$ & $\%$ \\
\hline \multicolumn{9}{|l|}{ Sexo } \\
\hline Hombres & 184 & 96.84 & 324 & 82.65 & 533 & 79.91 & 143,964 & 87.07 \\
\hline Mujeres & 6 & 3.16 & 68 & 17.35 & 134 & 20.09 & 21,383 & 12.93 \\
\hline \multicolumn{9}{|l|}{ Grupo de edad } \\
\hline$<10$ años & 5 & 2.63 & 29 & 7.40 & 79 & 11.84 & 11,686 & 7.07 \\
\hline 10-19 años & 20 & 10.53 & 101 & 25.77 & 160 & 23.99 & 74,133 & 44.83 \\
\hline 20-29 años & 17 & 8.95 & 68 & 17.35 & 144 & 21.59 & 32,524 & 19.67 \\
\hline 30-39 años & 19 & 10.00 & 60 & 15.31 & 104 & 15.59 & 14,825 & 8.97 \\
\hline 40-49 años & 37 & 19.47 & 42 & 10.71 & 70 & 10.49 & 11,937 & 7.22 \\
\hline 50-59 años & 32 & 16.84 & 42 & 10.71 & 51 & 7.65 & 4,974 & 3.01 \\
\hline 60 y mas & 59 & 31.05 & 50 & 12.76 & 59 & 8.85 & 15,270 & 9.23 \\
\hline No especificado & 1 & 0.53 & & & & & & \\
\hline \multicolumn{9}{|l|}{ Aseguramiento médico } \\
\hline No asegurado & 52 & 27.37 & 98 & 25.00 & 335 & 50.22 & 34,162 & 20.66 \\
\hline Asegurado & 111 & 58.42 & 244 & 62.24 & 289 & 43.33 & 131,186 & 79.34 \\
\hline No especificado & 27 & 14.21 & 50 & 12.76 & 43 & 6.45 & & \\
\hline \multicolumn{9}{|l|}{ ¿Habla lengua indígena? } \\
\hline Si & 19 & 10.00 & 14 & 3.57 & \multicolumn{2}{|c|}{$N . D$} & 2,968 & 1.80 \\
\hline No & 124 & 65.26 & 375 & 95.66 & & & 161,413 & 97.62 \\
\hline No especificado & 47 & 24.74 & 3 & 0.77 & & & 967 & 0.58 \\
\hline \multicolumn{9}{|l|}{ Estado civil } \\
\hline Soltero & 47 & 24.74 & \multicolumn{2}{|c|}{ N.D. } & \multicolumn{2}{|c|}{ N.D. } & 63,059 & 38.14 \\
\hline Viudo & 14 & 7.37 & & & & & 3,017 & 1.82 \\
\hline Separado & 5 & 2.63 & & & & & 5,433 & 3.29 \\
\hline Divorciado & 8 & 4.21 & & & & & 238 & 0.14 \\
\hline Union Libre & 30 & 15.79 & & & & & 10,759 & 6.51 \\
\hline Casado & 73 & 38.42 & & & & & 40,498 & 24.49 \\
\hline No aplica* & 7 & 3.68 & & & & & 42,343 & 25.61 \\
\hline No especificado & 6 & 3.16 & & & & & & \\
\hline \multicolumn{9}{|l|}{ Escolaridad } \\
\hline Menos de primaria & 88 & 46.32 & \multicolumn{2}{|c|}{ N.D. } & 44 & 6.60 & 58,889 & 35.62 \\
\hline Primaria completa & 49 & 25.79 & & & 265 & 39.73 & 41,044 & 24.82 \\
\hline Secundaria completa & 31 & 16.32 & & & 196 & 29.39 & 38,838 & 23.49 \\
\hline Bachillerato/Preparatoria completa & 6 & 3.16 & & & 76 & 11.39 & 10,300 & 6.23 \\
\hline Profesional o más & 2 & 1.05 & & & 19 & 2.85 & 11,879 & 7.18 \\
\hline No aplica** & 2 & 1.05 & & & 38 & 5.70 & 4,397 & 2.66 \\
\hline No especificado & 12 & 6.32 & & & 29 & 4.35 & & \\
\hline \multicolumn{9}{|l|}{ ¿Contra qué colisionó? } \\
\hline Contra motocicleta & 3 & 1.58 & 54 & 13.78 & 85 & 12.74 & \multirow{4}{*}{\multicolumn{2}{|c|}{ N.D. }} \\
\hline Contra automóvil o camioneta & 68 & 35.79 & 232 & 59.18 & 325 & 48.73 & & \\
\hline Contra transporte pesado o autobús & 17 & 8.95 & 33 & 8.42 & 27 & 4.05 & & \\
\hline No especificado & 102 & 53.68 & 73 & 18.62 & 230 & 34.48 & & \\
\hline
\end{tabular}

${ }^{*}$ No aplica en menores de 12 años.

${ }^{*}$ No se registró en menores de 6 años.

N.D.: No disponible; SIS-17: SIS-SS-17-P Hoja de Registro de Atención por Violencia y/o Lesión.

La gran mayoría de los lesionados egresó del hospital por mejoría $(79.34 \%)$; el $7.91 \%$ fue referido a otro hospital para su atención y solo 22 lesionados fallecieron en el hospital. En promedio, los ciclistas fueron hospitalizados 6.33 días (desviación estándar de 8.72), con un rango de 0 a 74 días de estancia hospitalaria, aunque el $32.14 \%$ de los pacientes estuvieron hospitalizados solo un día, el $12.50 \%$ dos días y el $10.46 \%$ tres días.

También la cabeza fue la región más afectada en las personas hospitalizadas, reportándose en el $32.14 \%$ de los casos. En poco menos del 17\% fueron la rodilla y la pierna la región más afectada, y en el $11.73 \%$ hubo «múltiples lesiones»3. 
Tabla 3. Región anatómica de la afección principal de ciclistas lesionados por el tránsito en México

\begin{tabular}{|c|c|c|c|c|c|c|c|c|}
\hline \multirow[t]{2}{*}{ Región anatómica } & \multicolumn{2}{|c|}{$\begin{array}{l}\text { Mortalidad } 2014 \\
\quad(\mathrm{~N}=190)\end{array}$} & \multicolumn{2}{|c|}{$\begin{array}{l}\text { Egresos } 2014 \\
\qquad(\mathrm{~N}=392)\end{array}$} & \multicolumn{2}{|c|}{$\begin{array}{l}\text { SIS-17 } 2014 \\
(\mathrm{~N}=667)\end{array}$} & \multicolumn{2}{|c|}{$\begin{array}{l}\text { ENSANut 2012* } \\
(\mathrm{N}=165,348)\end{array}$} \\
\hline & $\mathbf{N}$ & $\%$ & $\mathbf{N}$ & $\%$ & $\mathbf{N}$ & $\%$ & $\mathbf{N}$ & $\%$ \\
\hline Cabeza & 120 & 63.16 & 126 & 32.14 & 159 & 23.84 & 39,334 & 23.79 \\
\hline Cuello & 3 & 1.58 & 6 & 1.53 & 39 & 5.85 & & \\
\hline Tórax & 23 & 12.11 & 9 & 2.30 & 20 & 3.00 & 27,953 & 16.91 \\
\hline Abdomen, columna y pelvis & 10 & 5.26 & 19 & 4.85 & 20 & 3.00 & & \\
\hline Hombro y brazo & & & 25 & 6.38 & 38 & 5.70 & 63,462 & 38.38 \\
\hline Codo y antebrazo & & & 24 & 6.12 & 38 & 5.70 & & \\
\hline Muñeca y mano & & & 9 & 2.30 & 25 & 3.75 & 28,639 & 17.32 \\
\hline Cadera y muslo & 1 & 0.53 & 37 & 9.44 & 13 & 1.95 & 64,228 & 38.84 \\
\hline Rodilla y pierna & 1 & 0.53 & 65 & 16.58 & 64 & 9.60 & & \\
\hline Tobillo y pie & & & 7 & 1.79 & 54 & 8.10 & 29,213 & 17.67 \\
\hline Múltiples regiones & 22 & 11.58 & 46 & 11.73 & 179 & 26.84 & N.D. & N.D. \\
\hline Región no especificada & 5 & 2.63 & 11 & 2.81 & 16 & 2.40 & 16,917 & 10.23 \\
\hline $\begin{array}{l}\text { Cuerpo extraño por orificio } \\
\text { natural }\end{array}$ & 1 & 0.53 & & & & & $N . D$. & $N . D$. \\
\hline Ninguna (valor perdido) & 4 & 2.11 & 8 & 2.04 & 2 & 0.30 & 22,921 & $13.86^{* *}$ \\
\hline
\end{tabular}

${ }^{*}$ Corresponde al número de individuos que reportaron al menos una lesión en cada región anatómica de interés. Dado que los individuos pudieron tener múltiples lesiones, el total no corresponde necesariamente a la suma de las columnas previas y su suma puede exceder el número de ciclistas analizados.

**El tipo de lesión y su región anatómica no fue documentado en menores de 10 años $(N=11,686)$

N.D.: No disponible; SIS-17: SIS-SS-17-P Hoja de Registro de Atención por Violencia y/o Lesión.

De acuerdo con el análisis del Registro de Atención por Violencia o Lesión SIS-SS-17-P, en 2014 se registraron 667 casos de ciclistas lesionados $(1.53 \%$ del total de LCT), mayormente hombres (79.91\%) y que colisionaron contra un automóvil o una camioneta (48.73\%). Aunque el promedio de edad fue de 29.73 años (desviación estándar de 18.61), los adolescentes fueron el grupo más afectado (23.99\%), seguidos de los grupos de 20 a 29 años (21.59\%) y de 30 a 39 años (15.59\%). Un total de 32 ciclistas refirió tener una discapacidad preexistente $(4.80 \%)$, cuatro mujeres entre 9 y 59 años de edad estaban embarazadas al momento de la colisión y una de ellas sufrió un aborto como consecuencia.

Con respecto a la exposición a factores protectores y de riesgo, en menos del $1 \%$ se reportó que utilizaba el casco al momento de la colisión (0.75\%). Además, el $10.34 \%$ de los ciclistas fueron reportados bajo los efectos del alcohol y el $0.15 \%$ habían consumido otras drogas. En cuanto a la atención médica, solo un bajo porcentaje recibió atención médica prehospitalaria $(23.09 \%)$. El tiempo promedio transcurrido desde el inicio de la atención médica prehospitalaria y su llegada a una unidad hospitalaria fue de 140.93 minutos (desviación estándar de 551.08), con una mediana de 30 minutos y un rango de 5 a 5949 minutos o poco más de 4 días. En promedio, los lesionados estuvieron en el servicio de urgencias 119 minutos, prácticamente 2 horas. El servicio de urgencias fue el principal servicio que otorgó la atención a los lesionados (88.76\%), seguido del servicio de hospitalización $(6.60 \%)$ y del de consulta externa (3.45\%).

«Múltiples regiones» se registró como la región anatómica afectada con mayor frecuencia (26.84\%), seguida de la cabeza (23.84\%) y la rodilla y la pierna (9.60\%). Según muestra la tabla 4 , el uso de casco parece no estar asociado a la presencia de lesiones en la cara, el cráneo o el cuello, aunque es importante hacer notar el bajo número de lesionados que reportaron uso de casco.

De los datos de la ENSANut-2012 fueron identificados 117 ciclistas, que ponderados representan 165,348; el $12.6 \%$ del total de LCT (intervalo de confianza al 95\%: 9.8-16.2\%). La mayoría fueron hombres (87.07\%), y los adolescentes fueron el grupo de edad más afectado (44.83\%), seguidos de los de 20 a 29 años (19.67\%). La media de edad fue de 29.26 años, con un intervalo de confianza al $95 \%$ de 29.00 a 29.52. La 
Tabla 4. Uso de casco y lesiones de cabeza, cuello y cara en ciclistas lesionados por el tránsito en México.

\begin{tabular}{|c|c|c|c|c|c|}
\hline \multirow[t]{3}{*}{ Daños a la salud, por fuente de información } & \multicolumn{4}{|c|}{ Uso de casco } & \multirow[t]{3}{*}{ Valor $\mathbf{P}$} \\
\hline & \multicolumn{2}{|c|}{ No } & \multicolumn{2}{|c|}{ Sí } & \\
\hline & $\mathbf{N}$ & $\%$ & $\mathbf{N}$ & $\%$ & \\
\hline $\begin{array}{l}\text { SIS-17 (N = 665) } \\
\text { Otras lesiones } \\
\text { Lesión en cara } \\
\text { Lesión en cráneo } \\
\text { Lesión en cuello }\end{array}$ & $\begin{array}{c}482 \\
34 \\
124 \\
20\end{array}$ & $\begin{array}{c}73.03 \\
5.15 \\
18.79 \\
3.03\end{array}$ & $\begin{array}{l}4 \\
0 \\
1 \\
0\end{array}$ & $\begin{array}{c}80.00 \\
0.00 \\
20.00 \\
0.00\end{array}$ & $1.000^{*}$ \\
\hline $\begin{array}{l}\text { ENSANut-2012** }(N=153,662) \\
\text { Sin lesión en cabeza, cara o cuello } \\
\text { Con lesión en cabeza, cara o cuello }\end{array}$ & $\begin{array}{l}98,144 \\
39,334\end{array}$ & $\begin{array}{l}71.40 \\
28.60\end{array}$ & $\begin{array}{c}16,183 \\
0\end{array}$ & $\begin{array}{c}100.00 \\
0.00\end{array}$ & 0.239 \\
\hline
\end{tabular}

*Corresponde al valor $\mathrm{P}$ asociado a la prueba exacta de Fisher.

**El tipo de lesión y su región anatómica no fue documentado en menores de 10 años $(\mathrm{N}=11,686)$, por lo que no se incluyeron en este cuadro.

SIS-17: SIS-SS-17-P Hoja de Registro de Atención por Violencia y/o Lesión; ENSANut 2012: Encuesta Nacional de Salud y Nutrición 2012.

mediana en la muestra sin ponderar fue de 19 años. Según el estrato de residencia de los lesionados, el $64.60 \%$ viven en una metrópoli (con 100,000 o más habitantes), el $21.96 \%$ en zonas urbanas (con 100,000 o más y menos de 100,000 habitantes) y solo el $13.44 \%$ en zonas rurales (menos de 2500 habitantes).

$\mathrm{El}$ análisis de los factores protectores y de riesgo muestra que, de los ciclistas lesionados, solo el $5.98 \%$ sin ponderar y el $9.79 \%$ ponderado utilizaban casco al momento de lesionarse. Ninguno de los lesionados que utilizó casco reportó lesiones en la cabeza, comparado con el $28.60 \%$ de los que no usaban casco, aunque estas diferencias no fueron estadísticamente significativas, influido probablemente por el tamaño de la muestra (Tabla 4). El $7.62 \%$ de los lesionados refirieron ir bajo los efectos del alcohol al momento de la colisión. Recibió atención médica formal el $30.59 \%$ de los lesionados, el $30.07 \%$ se automedicó, el $5.70 \%$ recibió atención no formal y el $28.97 \%$ no recibió ningún tipo de atención.

Un total de 16,608 ciclistas reportaron consecuencias permanentes en su estado de salud como consecuencia de las LCT, la gran mayoría (60.81\%) relacionadas con limitaciones o dificultad para moverse o caminar (o bien hacerlo con ayuda), y el $15.35 \%$ refiere limitación o dificultad para usar sus brazos y manos. Las consecuencias permanentes están en estrecha relación con las regiones anatómicas más afectadas: el $38.84 \%$ tuvo afectación de miembros pélvicos, el $38.38 \%$ de miembros torácicos y el $23.79 \%$ de cabeza y cuello (Tabla 3 ).

\section{Discusión}

Hasta donde sabemos, este es el primer estudio llevado a cabo en México que documenta la epidemiología de las lesiones en ciclistas, unos de los usuarios más vulnerables de la vía pública. En general, se pudo observar que fue la cabeza una de las principales regiones anatómicas afectadas en estos usuarios viales, y los datos sugieren que puede estar relacionado con un peor desenlace en salud. Pareciera ser que el escaso uso de casco, particularmente de aquellos que cuentan con certificación, pudiera influir para que los traumatismos craneoencefálicos (TCE) fueran la lesión más común entre los ciclistas analizados. Aunque a partir de los datos analizados el casco parece no estar asociado a una menor incidencia de lesiones, es importante tomar en cuenta tres cosas: 1) el bajo número de ciclistas que reportaron utilizar casco al momento de las lesiones; 2) no hubo ciclistas lesionados en la ENSANut-2012 que reportaran alguna lesión en cara, cabeza o cuello; y 3) no se contó con información sobre si los cascos utilizados estaban certificados o no.

El uso de casco en ciclistas lesionados en México fue significativamente menor que lo reportado previamente en Alberta o Montreal(Canadá), donde el 26\% de las personas fallecidas y el $25 \%$ de los ciclistas que presentaron TCE, respectivamente, lo llevaban puesto ${ }^{13,14}$. El casco es una intervención dirigida a la protección del ciclista. En las colisiones de bicicletas intervienen dos mecanismos principales que causan TCE: el contacto directo y la aceleración y desaceleración, y cada mecanismo provoca distintas lesiones. Cuando ocurre una colisión en bicicleta, el ciclista por lo general es despedido del vehículo. Si la cabeza golpea un objeto, por ejemplo el suelo, se detiene el movimiento de la cabeza, pero el cerebro, que tiene su propia masa, continúa desplazándose hasta que golpea el lado opuesto del cráneo. Los resultados de este tipo de lesión son diversos, desde un TCE leve 
hasta un TCE grave que puede causar la muerte ${ }^{2}$. Los cascos aportan una capa adicional a la cabeza y, de ese modo, protegen de las formas más graves de $\mathrm{TCE}^{2}$, ya que según la evidencia disponible el uso de cascos para bicicleta reduce el riesgo de TCE entre un 63 y un $88 \%{ }^{15}$.

Para garantizar que los cascos prevengan efectivamente las lesiones de cráneo y cara deben cumplir con ciertos estándares de seguridad. Existen diferentes tipos de certificaciones en el mundo; por ejemplo, los cascos disponibles en los EE.UU. deben contar con la certificación CPSC (Consumer Products Safety Comission, que también avala las certificaciones ASTM F1447, F1898, Snell B-90A, B-95 y N-94) ${ }^{16}$; en la Unión Europea existen las certificaciones BS 6863, EN 1078:2012 y EN 1080:2013;y la certificación de Nueva Zelanda y Australia, conocida como AS/NZS $2063^{17}$. En México no existe una certificación para la fabricación y venta de cascos para ciclistas; mientras esto ocurre, debe recomendarse a los consumidores revisar el cumplimiento de alguna de estas certificaciones al momento de adquirir un casco ciclista.

Existe un debate sobre si legislar el uso obligatorio del casco, como han sugerido algunos autores ${ }^{18-20}$. Se considera una medida efectiva para incrementar su uso y disminuir el número de lesiones y de muertes en ciclistas $^{21}$. Otros autores difieren sobre esta medida, dado que desincentiva el uso de la bicicleta como medio de transporte ${ }^{22,23}$. Mientras este debate ocurre, un número importante de ciclistas se lesionan y mueren en el tránsito, por lo que es necesario empoderar a los usuarios de este medio de transporte con la evidencia disponible para que conozcan los riesgos a los que se enfrentan y los potenciales efectos preventivos del uso de casco.

La promoción del uso de casco, sin embargo, no debería ser la única, y menos la más importante, medida para prevenir lesiones en ciclistas. Es tan solo una medida que debe formar parte de un abordaje integral al problema, en el que se incluyan elementos clave como: a) el diseño de las vialidades que debe ser sensible a las necesidades de espacio de los ciclistas, lo que en vialidades primarias y secundarias supone comúnmente la construcción de ciclo-vías para separar a este usuario y evitar su contacto con vehículos motorizados ${ }^{7}$; b) la disminución de la velocidad de los vehículos motorizados, que puede lograrse mediante reductores de velocidad (topes, semáforos, etc.) o el establecimiento de límites de velocidad y su aplicación rigurosa, directamente o mediante la implementación de estrategias como la foto-infracción ${ }^{24} ; c$ ) la promoción de la bicicleta como medio de transporte, pues según algunos autores el aumento en el número de ciclistas en circulación es en sí mismo una forma de disminuir el riesgo de las lesiones, lo que es conocido como "seguridad en números" (safety in numbers) $)^{25} \mathrm{y}$; d) estrategias educativas tendentes a lograr conductas más seguras en todos los usuarios de la vía pública y el uso de dispositivos de seguridad (luces y reflectantes). Aquí sería importante promover entre los ciclistas el no utilizar la bicicleta bajo la influencia de alcohol y otras drogas, pues se observó que el 10\% de los lesionados captados en el SIS-17 y el $8 \%$ de Ios ciclistas lesionados de la ENSANut-2012 refirieron ir bajo los efectos del alcohol, aunque este nivel de exposición es menor a lo reportado en Alberta (Canadá) en ciclistas fallecidos en el tránsito $(25 \%)^{13}$. Todas estas medidas, en conjunto, podrían contribuir a prevenir el alto número de lesiones fatales y no fatales en estos usuarios vulnerables de la vía pública?.

Por otro lado, el alto porcentaje de ciclistas que fallecen en la vía pública $(63.16 \%)$, mayormente sin recibir asistencia médica $(89.17 \%)$, nos indica la gravedad de las lesiones sufridas. Esto destaca la importancia de crear las condiciones de seguridad necesarias para minimizar los daños a la salud producidos por este medio de transporte, toda vez que en la gran mayoría de los casos reportados (77.27\%) las colisiones ocurrieron contra un automóvil o camioneta, en franca desventaja. Este porcentaje es significativamente mayor quelo observado en contextos como Nueva Zelanda, donde el $26 \%$ de todas las lesiones en ciclistas y el $39 \%$ de las lesiones serias en ciclistas resultaron de una colisión contra este tipo de vehículos de motor ${ }^{26}$. En este mismo sentido destaca el bajo número de ciclistas lesionados cubiertos por los servicios médicos prehospitalarios (23\%), según el registro SIS-17, lo que en conjunto es un llamado urgente a mejorar la regulación de este tipo de atención y la conformación de un Sistema Médico de Emergencias en el país, tarea aún pendiente ${ }^{27}$.

Este estudio tiene diversas limitaciones, mayormente atribuidas al hecho de que las fuentes de información utilizadas fueron secundarias. Por un lado, es importante señalar la necesidad de mejorar los sistemas de información para incluir la recolección sistemática de información sobre factores de riesgo y factores protectores que permita informar la toma de decisiones y el diseño de políticas públicas de seguridad vial, así como evaluar el impacto de intervenciones específicas. En este sentido, no se recabó el uso de casco en el $71.21 \%$ de los lesionados registrados por el SIS-17. Por otro 
lado, el diseño de la ENSANut 2012 buscó representatividad nacional para eventos con una prevalencia del $10 \%{ }^{28}$. Mientras que la prevalencia de ciclistas lesionados pudiera ser un indicador relativamente confiable, las características sociodemográficas presentadas en este trabajo pudieran no ser representativas del total de los ciclistas que sufrieron lesiones no fatales durante 2012 en el país. Sin embargo, esta es la información más confiable hasta el momento, particularmente porque incluye a las personas lesionadas que no acudieron a los servicios de salud por problemas en el acceso o por poca gravedad de sus lesiones. Información de utilidad, como la certificación de los cascos, no estaba presente. De ahí que sean necesarios otros abordajes metodológicos para evaluar si realmente los cascos no ofrecen protección, si esto es debido a que los que se están utilizando no están certificados o si existen otros factores que lo expliquen. De igual forma, los resultados podrían verse enriquecidos con información sobre el uso de casco en ciclistas mediante la observación de este usuario vialen distintos contextos del país (información no disponible al momento). Esto permitiría conocer si los ciclistas que usan casco asumen conductas más seguras en México o si, por el contrario, la percepción de protección que les ofrece el casco los hace adoptar conductas riesgosas, tal como ha sido documentado en otros contextos ${ }^{29}$.

Podemos concluir que si bien al momento los ciclistas no representan un grupo proporcionalmente importante, al existir distintas estrategias para incentivar el uso de la bicicleta como medio de transporte sostenible es necesario anticiparse para prevenir los potenciales efectos de estas estrategias en términos de LCT. Para ello, mientras se garantizan vialidades seguras en el mediano y largo plazo, como uno de los principales factores asociados a la seguridad de los ciclistas, deben intensificarse las medidas de control de la velocidad, el uso de luces y reflectores y particularmente la promoción del uso del casco certificado en el corto plazo, pues es una medida también efectiva para prevenir el principal tipo de lesión que enfrentan los ciclistas: los TCE. Al no existir análisis previos que integren todas estas fuentes de datos, este trabajo sentará la base que permitirá realizar análisis más detallados para entender mejor los daños a la salud asociados a este medio de transporte sostenible.

\section{Agradecimientos}

Los autores desean agradecer al equipo del Instituto de Políticas para el Transporte y el Desarrollo que contribuyó a la elaboración del documento Más ciclistas, más seguros: guía de intervenciones para la prevención de lesiones en ciclistas que a través de distintas discusiones académicas estimuló la realización de este estudio buscando aportar evidencia para futuros análisis y nuevas discusiones.

\section{Conflictos de intereses}

Los autores declaramos no tener ningún conflicto de intereses.

\section{Bibliografía}

1. World Health Organization. Global Status Report on Road Safety 2015. Geneva, Switzerland: WHO; 2015. Disponible en: http://www.who.int/ violence_injury_prevention/road_safety_status/2015/en/.

2. Organización Panamericana de la Salud. Cascos. Manual de seguridad vial para decisores y profesionales. Washington, D.C., EUA: OPS; 2008. Disponible en: http://www.bvsde.paho.org/bvsdevial/fulltext/Cascos/indice.pdf.

3. Sistema de Transporte Individual ECOBICI. Ciudad de México: Secretaría del Medio Ambiente.(Accedido el 20 de mayo de 2016.) Disponible en: https://www.ecobici.df.gob.mx.

4. Sistema de Bici Pública MIBICl. Guadalajara: Secretaría de Movilidad. (Accedido el 20 de mayo de 2016.) Disponible en: https://mibici.net/a-9meses-de-mibici/.

5. SmartBike. Puebla: H. Ayuntamiento de Puebla. (Accedido el 20 de mayo de 2016.) Disponible en: http://www.pueblacapital.gob.mx/noticias/ itemlist/category/314-smartbike.

6. Sistema de Bici Pública Huizi. Toluca: H. Ayuntamiento de Toluca. (Accedido el 20 de mayo de 2016.) Disponible en: https://www.facebook. com/BiciPublicaToluca/ (ya no hay otra página oficial que anide el sitio, sólo Facebook).

7. Secretaría de Salud/STCONAPRA/ITDP. Más ciclistas, más seguros. Guía de intervenciones para la prevención de lesiones en ciclistas urbanos. Ciudad de México, México; 2016.(Accedido el 20 de mayo de 2016.) Disponible en: https://www.gob.mx/cms/uploads/attachment/file/83621/M_s_ciclistas ITDP final carta electr nico .pdf.

8. Dirección General de Epidemiología, Secretaría de Salud Federal. Subsistema Epidemiológico y Estadístico de Defunciones (SEED). México, 2014. (Accedido el 20 de mayo de 2016.) Disponible en: http://www.dgis. salud.gob.mx/contenidos/basesdedatos/std_defunciones gobmx.html.

9. Dirección General de Información en Salud, Secretaría de Salud Federal. Subsistema Automatizado de Egresos Hospitalarios (SAEH). México; 2014. (Accedido el 20 de mayo de 2016.) Disponible en: http:/l www.dgis.salud.gob.mx/contenidos/basesdedatos/std_egresoshospitalarios_gobmx.html

10. Dirección General de Información en Salud, Secretaría de Salud Federal. Registro de Atención por Violencia y/o Lesión (SIS-17).México; 2014. (Accedido el 20 de mayo de 2016.) Disponible en: http://www.dgis.salud. gob.mx/contenidos/basesdedatos/da_lesiones_gobmx.html.

11. Instituto Nacional de Salud Pública. Encuesta Nacional de Salud y Nutrición 2012. Resultados Nacionales. México; 2012.(Accedido el 20 de mayo de 2016.) Disponible en: http://ensanut.insp.mx/basesdoctos.php\#. V47VavnhBhE.

12. Organización Panamericana de la Salud. Clasificación Estadística Internacional de Enfermedades y Problemas Relacionados con la Salud. Décima revisión. 10. ${ }^{\text {a }}$ ed. Washington, DC: OPS; 1995.

13. Gaudet L, Romanow NTR, Nettel-Aguirre A, et al. The epidemiology of fatal cyclists crashes over a 14-year period in Alberta, Canada. BMC Public Health. 2015;15:1142.

14. Dagher JH, Costa C, Lamoureux J, et al. Comparative outcomes of traumatic brain injury form biking accidents with or without helmet use. Can J Neurol Sci. 2016;43:56-64.

15. Peden MM, Scurfield R, Mohan D, et al, editores. World report on road traffic injury prevention. Geneva: World Health Organization; 2004.(Accedido el 20 de mayo de 2016.)Disponible en: http://www.who.int/violence_injury_prevention/publications/road_traffic/world_report/en/.

16. Consumer Products Safety Comission. EUA: Which helmet for which activity?(Accedido el 20 de mayo de 2016.)Disponible en: https://www. cpsc.gov/safety-education/safety-guides/sports-fitness-and-recreation-bicycles/which-helmet-which-activity.

17. Product Safety Australia.Australia: Bicycle helmets. (Accedido el 20 de mayo de 2016.)Disponible en: http://www.productsafety.gov.au/content/ index.phtml/tag/bicyclehelmets. 
18. Walter SR, Oliver J, Churches T, et al. The impact of compulsory helmet legislation on cyclist head injuries in New South Wales, Australia: aresponse. Accid Anal Prev. 2013;52:204-9

19. Macpherson A, Spinks A.Bicycle helmet legislation for the uptake of helmet use and prevention of head injuries. Cochrane Database Syst Rev. 2008;(3):CD005401

20. Karkhaneh M, Kalenga JC, Hagel BE, et al.Effectiveness of bicycle helmet legislation to increase helmet use: a systematic review. Inj Prev.2006;12:76-82.

21. Persaud N, Coleman E, Zwolakowski D, et al.Nonuse of bicycle helmets and risk fatal head injury: a proportional mortality, case-control study. CMAJ. 2012;184:E921-3.

22. Robinson DL. No clear evidence from countries that have enforced the wearing of helmets. BMJ. 2006;332:722-5.

23. Cycling UK.Cycle helmets: an overview of the evidence. London: Cycling UK; 2016. (Accedido el 20 de julio de 2016.) Disponible en: http://www. cyclinguk.org/sites/default/files/file_public/helmets-evidencebrf.pdf.

24. Elvik R. Quantified road safety targets: an assessment of evaluation. Oslo: Institute of Transport Economics; 2001 Oct. TØI Report 539/2001.
(Accedido el 20 de julio de 2016.)Disponible en: https://www.toi.no/getfile.php?mmfileid=17511.

25. Jacobsen PL.Safety in numbers: more walkers and bicyclists, safer walking and bicycling. Inj Prev. 2003;9:205-9

26. Tin-Tin S, Woodward A, Ameratunga S. Injuries to pedal cyclists on New Zealand roads, 1988-2007. BMC Public Health. 2010;10:655.

27. NORMA Oficial Mexicana NOM-034-SSA3-2013, Regulación de los servicios de salud. Atención médica prehospitalaria. Diario Oficial de la Federación. Organo del Gobierno Constitucional de los Estados Unidos Mexicanos, 23 de septiembre 2014; número 17, sección I, Tomo DCCXXXII.(Accedido el 20 de mayo de 2016.) Disponible en: http://dof.gob.mx/nota_detalle.php?codigo $=5361072 \& f e c h a=$ 23/09/2014.

28. Romero-Martínez M, Shamah-Levy T, Franco-Núñez A, et al. Encuesta Nacional de Salud y Nutrición 2012:diseño y cobertura. Salud Publica Mex. 2013;55(Supl 2):S332-40.

29. Gamble T, Walker I. Wearing a bicycle helmet can increase risk taking and sensation seeking in adults. Psychol Sci. 2016;27:289-94. 